\title{
Examining the Expectation Deficit in Normal Aging
}

\author{
Hannah L. Pincham, Clare Killikelly, Laura Vuillier, and Alan J. Power \\ Department of Experimental Psychology, University of Cambridge, Cambridge CB2 3EB, United Kingdom \\ Review of Zanto et al.
}

A frequently examined ability that deteriorates with age is attentional orienting (Hämmerer et al., 2010; Bollinger et al., 2011). Orienting one's attention toward relevant information helps to optimize performance, but older adults typically show an impaired use of predictive cues to guide attention (Hämmerer et al., 2010; Bollinger et al., 2011). Consistent with theories that attribute age-related decline to decreased frontal lobe connectivity $(\mathrm{Fa}-$ biani and Gratton, 2012), functional magnetic resonance imaging (fMRI) indicates that older adults' attentional orienting deficit is underpinned by diminished functional connectivity between the prefrontal cortex and the visual association cortex (Bollinger et al., 2011).

Research in young adults has suggested that the ability to anticipate the timing of upcoming events is also imperative for optimal behavior (Stefanics et al., 2010). However, before a recent publication by Zanto et al. (2011) in The Journal of Neuroscience, older adults' facility with temporal orienting had not been explored. Because of its high temporal resolution relative to fMRI, electroencephalography (EEG) is a crucial tool for studying temporal orienting. Responding to the absence of age-related temporal orienting research, Zanto et al. (2011) used EEG to

Received Oct. 18, 2011; revised Nov. 22, 2011; accepted Nov. 23, 2011. We thank Gábor Stefanics for his useful comments on an earlier version of this manuscript.

Correspondence should be addressed to Hannah Pincham, Department of Experimental Psychology, University of Cambridge, Downing Street, Cambridge CB2 3EB, UK. E-mail: hlp31@cam.ac.uk.

DOI:10.1523/JNEUROSCI.5255-11.2012

Copyright $\odot 2012$ the authors $\quad 0270-6474 / 12 / 321143-03 \$ 15.00 / 0$ compare temporal orienting across older and younger adults.

Zanto et al. (2011) tested younger (mean age 24.6 years) and older (mean age 69.7 years) adults on a temporally cued target-response task while recording participants' ongoing EEG. Predictive (P) cue letters signaled the length of the cuetarget foreperiod, which was either short (S, $600 \mathrm{~ms}$ ) or long (L, $1400 \mathrm{~ms}$ ). Neutral (N) cues were equally followed by long or short foreperiods, thus creating four possible trial types: PS, PL, NS, NL.

Younger and older adults' performance was compared using four-way ANOVAs with task complexity, foreperiod (short, long), cue (predictive, neutral) and age (older, younger) as factors. This analysis was applied to participants' reaction time (RT) data, the visual eventrelated potentials (ERPs) evoked by the cue itself (P1 and N1), the contingent negative variation $(\mathrm{CNV})$, and alpha power during the final $200 \mathrm{~ms}$ of the foreperiod, and finally the ERPs evoked by the target stimulus (P1, N1, and P3).

The major finding was that younger, but not older, adults used predictive information to improve task performance. This improvement was reflected in faster RTs to PS versus NS targets. Additionally, CNV amplitude (a neural measure of expectancy) was more negative in the foreperiod following PS than that following NS cues in younger adults only, indicating that the preparatory benefit of the PS cue was restricted to younger adults. Younger adults also showed larger alpha power modulation in the foreperiod, suggesting that preparatory neural mechanisms were more efficient in the frequency bands comprising the CNV and alpha activity in this age group. Although older adults showed some modulation of alpha power in the short foreperiod, the magnitude of modulation was significantly greater for young adults. The absence of RT or alpha power differences between PL and NL trials (across both younger and older adults) suggested that there was no effect of cueing for the long foreperiod. However, one would not expect differences in the long foreperiod because the predictive benefit derived from PL cues cannot exceed that provided by conditional probabilities; if the target is not displayed after a short foreperiod, it must occur after the long foreperiod.

Based on these results, Zanto et al. (2011) concluded that older adults are unable to engage the neural mechanisms underlying temporal cueing and therefore cannot improve their performance on this task. The authors argued that these findings provide support for a more general "expectation deficit" hypothesis, which posits that older adults fail to derive benefit from the forewarning of expected events, as a result of inadequate expectation-based neural mechanisms. Although Zanto et al.'s conclusions are justified by their data, alternative interpretations might add value to those conclusions.

It has been shown that older adults suffer a deficit in goal maintenance (Paxton et al., 2008). Specifically, impaired goal maintenance is related to compensatory shifts in older adults from proactive cognitive control (used by younger adults) to 
reactive cognitive control. When proactive control is engaged, goal-relevant information is actively maintained in an anticipatory manner to optimally bias attention (Braver et al., 2009). Therefore, Zanto et al.'s (2011) results could be interpreted as evidence for a general shift to reactive control rather than a specific deficit in impaired temporal expectation. In other words, younger adults may manage the PS cue-target combination using proactive control, whereas older adults rely on reactive control. Furthermore, Zanto et al. cite the top-down influence of frontoparietal networks over alpha and CNV activity and suggest that the aging deficit may stem from deterioration of frontoparietal attentional networks. Given the influence of these networks, the possible involvement of reactive control is bolstered by the fact that the prefrontal cortex is implicated in older adults' switch to reactive control (Paxton et al., 2008). Accordingly, although Zanto et al.'s results convincingly implicate impaired expectation in older adults, further work considering the possibility of a more general overarching deficit in goal maintenance and proactive versus reactive control may help to clarify the primary mechanism driving impaired expectation.

Successful cue-target responding is a complex process that demands success at multiple substages of information processing, including visual perception, extraction of cue meaning, and response preparation (Fig. 1). Although Zanto et al. (2011) position the locus of the older adults' behavioral deficit at the level of expectation or preparation (Fig. 1C), it is possible that the deficit is also present earlier in the processing stream. Zanto et al. suggest that nonsignificant correlations between the amplitude or latency of the cue-N1 and the amplitude of the CNV or alpha power excludes early visual processing delays (Fig. 1A) as the cause of the older adults' deficit. However, the current data cannot exclude age-related differential cue processing more generally. This is because the significant age-foreperiod (PS and NS vs PL and NL) interaction for the cue-P1 must have been driven by differential processing of the PS and PL cues. The neutral cues $(\mathrm{N})$ were indistinguishable at the time of cue onset, such that the significant age-foreperiod effect could only have been driven by age-related differences in neural activity between the two predictive cues.

Alternatively, the older adults may have experienced difficulty at a slightly later processing stage, while extracting

Attention Shifting

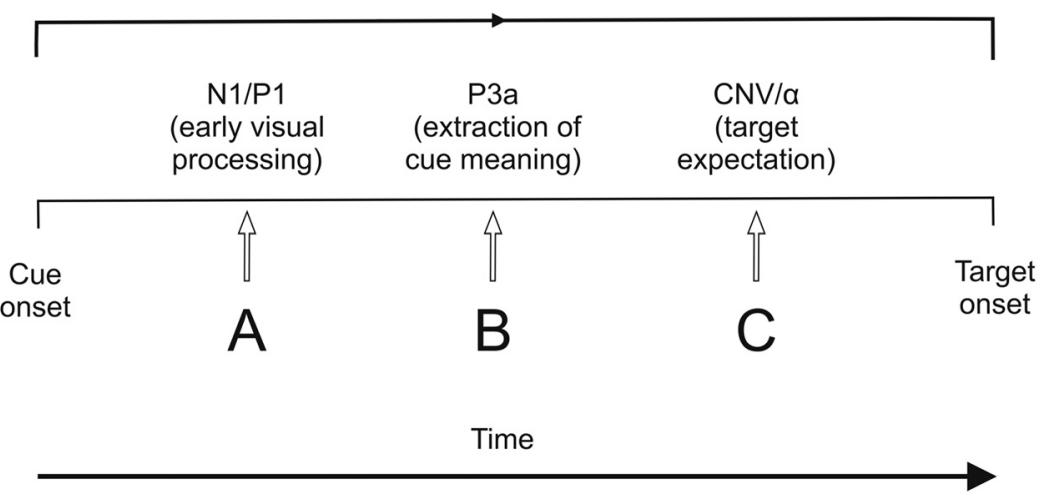

Figure 1. A subset of the stages involved in information processing in a cued target-response task. $A$, Following the onset of the cue, it must undergo visual sensory processing (indexed by the $\mathrm{N} 1$ and $\mathrm{P} 1$ event-related potential components). $\boldsymbol{B}$, Extraction of cue meaning can be electrophysiologically indexed by the P3a component. C, Target expectation or preparation proceeds the extraction of cue meaning, and can be indexed by the CNV or alpha power. Additionally, between the onset of the cue and that of the target, attention must be shifted from the cue to the target stimulus.

task-related information from the predictive cues and/or shifting their attention from cues to targets (Fig. $1 B$ ). Previous empirical work supports the idea that older adults have trouble extracting cue information and experience difficulty shifting their attentional focus, resulting in deficient response preparation (Hämmerer et al., 2010). An examination of the cue-P3a could help to disambiguate Zanto et al.'s (2011) findings, because the $\mathrm{P} 3 \mathrm{a}$ is indicative of lifespan changes in cue utilization (Fallgatter et al., 1999; Hämmerer et al., 2010; Tays et al., 2008). Specifically, Hämmerer et al. (2010) demonstrated that the frontal P3a is enhanced in older adults during a cued no-go task, and they argued that the increased contribution of frontal areas is indicative of greater attentional distraction costs resulting from inefficient discrimination between task-relevant and task-irrelevant cues. If future work revealed a relationship between the cue-P3a (as an index of cue utilization) and prestimulus $\mathrm{CNV}$ or alpha (indexing preparation/expectation), support would be provided for the differential utilization of cue meaning across age groups.

Regardless of the exact mechanism underpinning Zanto et al.'s (2011) results, these results could be usefully placed within the neural framework of diminished top-down control (Fabiani and Gratton, 2012). This framework, which asserts that behavioral deficits in older adults result from reduced connectivity in the neural circuits involved in top-down control, has been implicated in various investigations of age-dependent brainbased changes. Zanto et al. themselves refer to an age-related deficit in frontopa- rietal network activity. Correspondingly, the possible alternative interpretations put forward here would also fit into this framework. First, frontostriatal areas (specifically the dorsolateral prefrontal cortex) physiologically underlie proactive control. Paxton et al. (2008) suggest that older adults' shift from proactive to reactive control may be driven by a decline in the structural integrity of the prefrontal cortex. Second, changes in frontoparietal circuitry are implicated in older adults' difficulties with cue utilization and cuetarget shifting. Madden et al. (2010) report less functional connectivity within the frontoparietal neural circuit in older adults during cue processing in a cued task switching paradigm.

Although the exact cognitive and neural processes driving Zanto et al.'s (2011) results have yet to be established, Zanto et al. clearly document the temporal orienting deficit experienced by older adults and highlight the need to examine the temporal dimension of cognition within normal aging. Their results also help to advance an understanding of the neuro-cognitive basis of attentional orienting more generally. Future research exploring the contributions of proactive and reactive control, the cue-P3a, and frontally based neural networks that mediate top-down control should help to clarify this novel and exciting field.

\section{References}

Bollinger J, Rubens MT, Masangkay E, Kalkstein J, Gazzaley A (2011) An expectation-based memory deficit in aging. Neuropsychologia 49:14661475.

Braver TS, Paxton JL, Locke HS, Barch DM (2009) 
Flexibile neural mechanisms of cognitive control within human prefrontal cortex. Proc Natl Acad Sci U S A 106:7351-7356.

Fabiani M, Gratton G (2012) Aging, working memory, and attention control: a tale of two processing streams? In: Principles of frontal lobe function, Ed 2 (Stuss DT, Knight RT, eds). New York: Oxford UP. In press.

Fallgatter AJ, Mueller TJ, Strik WK (1999) Agerelated changes in the brain electrical correlates of response control. Clin Neurophysiol 110:833-838.

Hämmerer D, Li SC, Müller V, Lindenberger U (2010) An electrophysiological study of re- sponse conflict processing across the lifespan: Assessing the roles of conflict monitoring, cue utilization, response anticipation, and response suppression. Neuropsychologia 48: 3305-3316.

Madden DJ, Costello MC, Dennis NA, Davis SW, Shepler AM, Spaniol J, Bucur B, Cabeza R (2010) Adult age differences in functional connectivity during executive control. Neuroimage 52:643-657.

Paxton JL, Barch DM, Racine CA, Braver TS (2008) Cognitive control, goal maintenance, and prefrontal function in healthy aging. Cereb Cortex 18:1010-1028.
Stefanics G, Hangya B, Hernádi I, Winkler I, Lakatos P, Ulbert I (2010) Phase entrainment of human delta oscillations can mediate the effects of expectation on reaction speed. J Neurosci 30:13578-13585.

Tays WJ, Dywan J, Mathewson KJ, Segalowitz SJ (2008) Age differences in target detection and interference resolution in working memory: an event-related potential study. J Cogn Neurosci 20:2250-2262.

Zanto TP, Pan P, Liu H, Bollinger J, Nobre AC, Gazzaley A (2011) Age-related changes in orienting attention in time. J Neurosci 31: 12461-12470. 